\title{
Absolute Position Determination with Single Vision Using Laser Range Finders
}

\author{
H. S. Woo, K. S. Kwon, and B. G. Kim
}

\begin{abstract}
With the speed of information networks and the demand for real time service over the past few years, demand for up-to-date spatial information has accordingly been rising. Mobile mapping systems can be efficient equipments that meet such requirements to achieve real-time spatial information. For that, rapid absolute position determination is needed using images obtained from mobile mapping systems. A method has been proposed, which determine the absolute position of single vision in real time using sensor position, attitude information, and distances acquired from laser range finders. The proposed method provides the solution of absolute position with distances of more than 3 measuring points and angles between rays connecting the lens to the road surface. It is expected that the proposed method can perform rapid geospatial data generation from single vision by minimizing separate post-processing for absolute position determination.
\end{abstract}

Index Terms-Laser range finders, real time, absolute position, single vision.

\section{INTRODUCTION}

Geospatial mapping can be defined as the process of collecting geospatial data and it is one of the fundamental requirements in geo-informatic engineering. The primary results from the mapping process include absolute positioning, 3D object analysis, GIS data, georeferenced images and vedio and so on. For that, various remote sensing equipments such as optical sensors, radar, laser, LiDAR have been used.

Among them, mobile mapping employing a mobile vehicle to collect geospatial data have been used frequently to rapidly construct and update spatial information in recent years. The advent of mobile mapping system has generated a not inconsiderable ripple effect on the whole community of geo-informatic engineering and remote sensing in terms of real-time acquisition of geospatial information from remote sensing equipments. With the speed of information networks and demand for real time service increasing dramatically over the past few years, demand for up-to-date spatial information has accordingly been rising. Mobile mapping system can meet the requirements by facilitating real-time acquisition of absolute three dimensional position.

Manuscript received November 25, 2012 ; revised January 10, 2013. This research was supported by a grant from Cutting-Edge Urban Development-Korean Land Spatialization Research Project funded by Ministry of Land, Transport and Maritime Affairs (06KLSGB01) and by the supporting project to educate GIS experts funded by the Ministry of Land, Transport and Maritime Affairs of the Government of Korea.

The authors are with the Dept. of Geo-informatic Eng., Inha University, Incheon, Korea (e-mail: heesook@inha.edu; kskwon02@gmail.com; byungkim@inha.ac.kr).
Previous investigations have demonstrated the potential of mobile mapping system for real-time absolute positioning. However, in general, they require additional post-processing and the use of LiDAR or stereo images in order to enhance the precision of absolute coordinate determination. Jeong(2007) programmed and compared two space intersection methods used in photogrammetry to select more proper method for the three dimensional positioning in the field processing [1]. He employed the analytic space intersection to implement three dimensional positioning. $\mathrm{He}$ modified and applied to that to adapt to mobile mapping system's characteristics that camera position and attitude are changed according to the vehicle movement. Oh and Lee(2010) developed a method to determine the three dimensional coordinates of an object point from overlapping omnidirectional images acquired by a ground mobile mapping system [2]. They confirmed that the relative coordinates of the object point with the accuracy of several centimeters could be confirmed with the appropriate length of baseline and mutual positions between cameras and objects. Aly(2008) presented a robust approach to lane marker detection in urban streets [3]. They applied RANSAC Spline Fitting algorithm to refine the detected straight lines and correctly detect curved lanes and additionally performed post processing to enhance image matching performances. They could confirmed that the algorithm can detect all lanes in still images of urban streets and works at high rates. Touly et al.(2010) presents a novel real-time distance determination algorithm using an image sensor for use in an automobile environment, which is implemented in the $\mathrm{C}$ language using the OpenCV libraries [4]. It was observed that implementation in OpenCV leads to a high performance, low overhead system that could be implemented on a low power embedded device in an automotive environment. In addition, they confirmed that distance determination coupled with robust object detection routines could provide innovative and life saving systems to aid in driver safety and awareness. One study implemented the SLAM system using a single camera and a PSD sensors [5]. It detects obstacles from the front PSD sensor and then perceive size and feature of the obstacles by image processing. The probability SLAM was implemented using sensor data and image and the performance of the system was verified by real experiment.

Likewise, previous investigations have demonstrated potential of mobile mapping but they have mainly adapted additional post-processing or application of data to determine and enhance the precision of three dimensional absolute positioning. In this study, we propose a method to achieve absolute position from a single image by minimizing the addition steps. 


\section{Proposed Method}

Using laser range finders, we acquired distances of measuring points for the road surface as a horizontal reference plane. We extracted the relationship between the camera and the road surface and then determined the geometric attitude of the road surface. To determine absolute position from a single image based on the geometric attitude, we defined a virtual target reference plane assuming the road surface is flat and road sides are vertical planes as seen in Fig. 1.

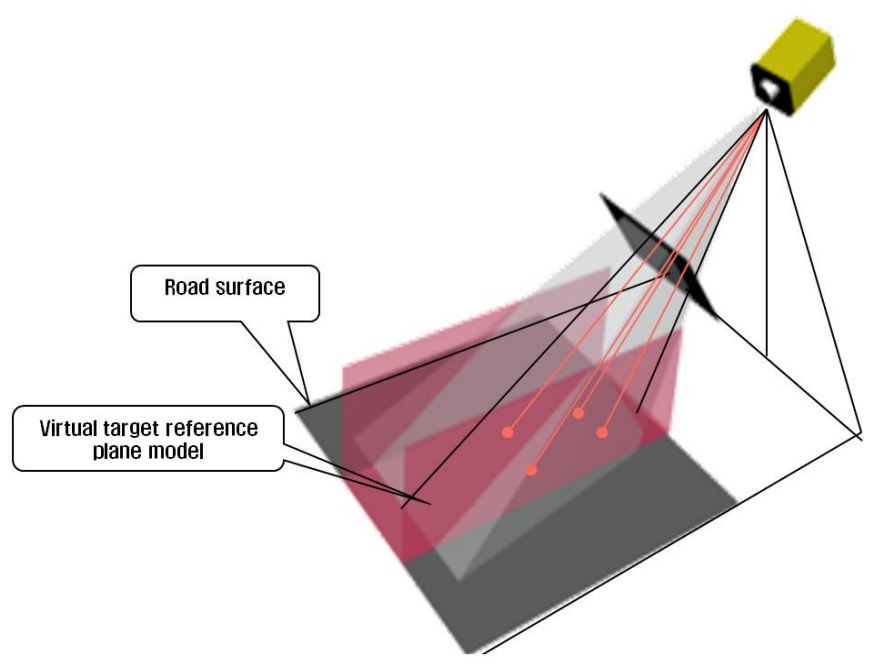

Fig. 1. Relationship between laser range finders and target reference plane.

When the virtual target reference plane is filmed assuming the road surface is flat, the road surface is projected on an image, as seen in Fig. 2. From the Fig. 2, we acquired $h$, distance from lens to surface, and $\mathrm{R}_{\text {front }}$ and $\mathrm{R}_{\text {back, }}$, distances from lens to measuring points, using laser range finders. If there are distances for more than 3 measuring points and angles between rays that connect the lens to the road surface, the solution for absolute positioning can be interpreted for the longitudinal $(X)$ and transverse $(Y)$ directions as seen in Fig. 3 and 4, respectively. This can be represented as equation (1). Through this relation, the absolute position can be determined from single vision.

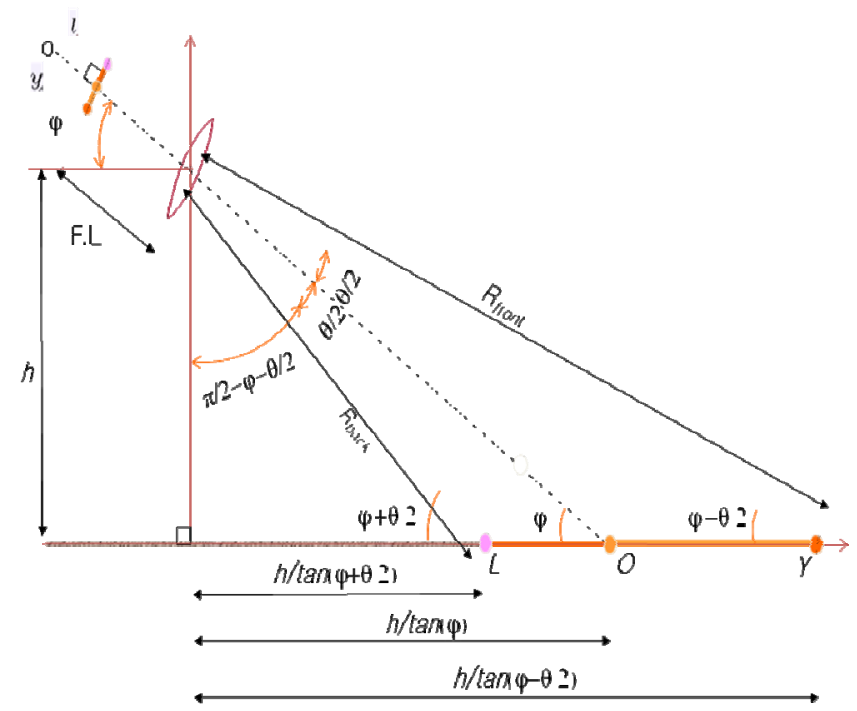

Fig. 2. Illustration of projection based on target reference plane model

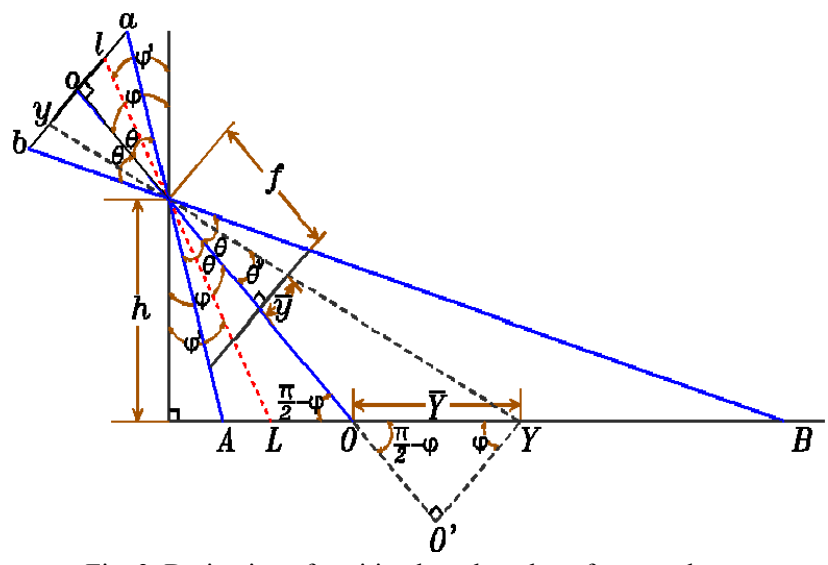

Fig. 3. Derivation of position based on the reference plane model(longitudinal direction)

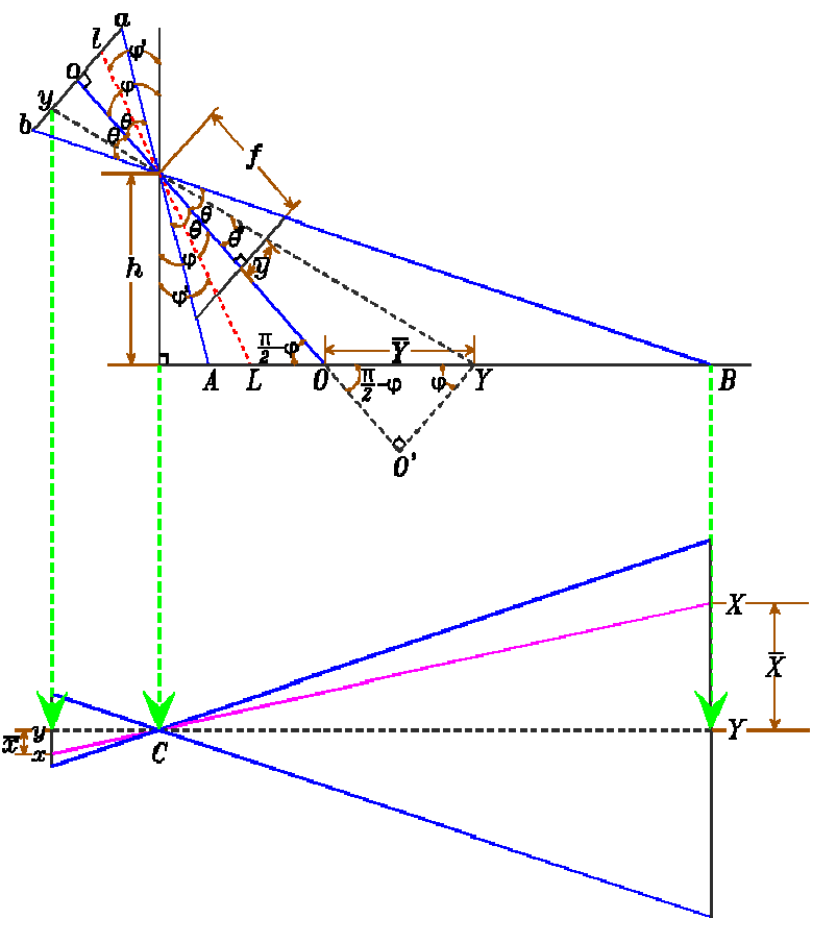

Fig. 4. Derivation of position based on the reference plane model(transverse direction)

Fig. 5 presents the procedure to achieve the absolute position from single vision in real time. First, inner orientation parameters are determined using camera information and exterior orientation parameters are determined through a laser pattern searched within acquired images. Meanwhile, the geometric relationship between the road surface and image is extracted using the sensor position, attitude information, and distances using laser range finders, and an image for a virtual reference plane model is thereby generated. Once exterior orientation parameters and geometric attitude of the reference plane are determined, image extraction is carried out from the target reference plane area. Absolute coordinates system is then determined for image extraction area. The absolute coordinate systems of the extraction area are decided, then the absolute position is determined directly within the image. The absolute positioning process also includes checking if the extracted absolute positions are valid coordinates to be suitable for 
target reference plane. Through the whole process, rapid absolute positioning can be implemented from single vision.

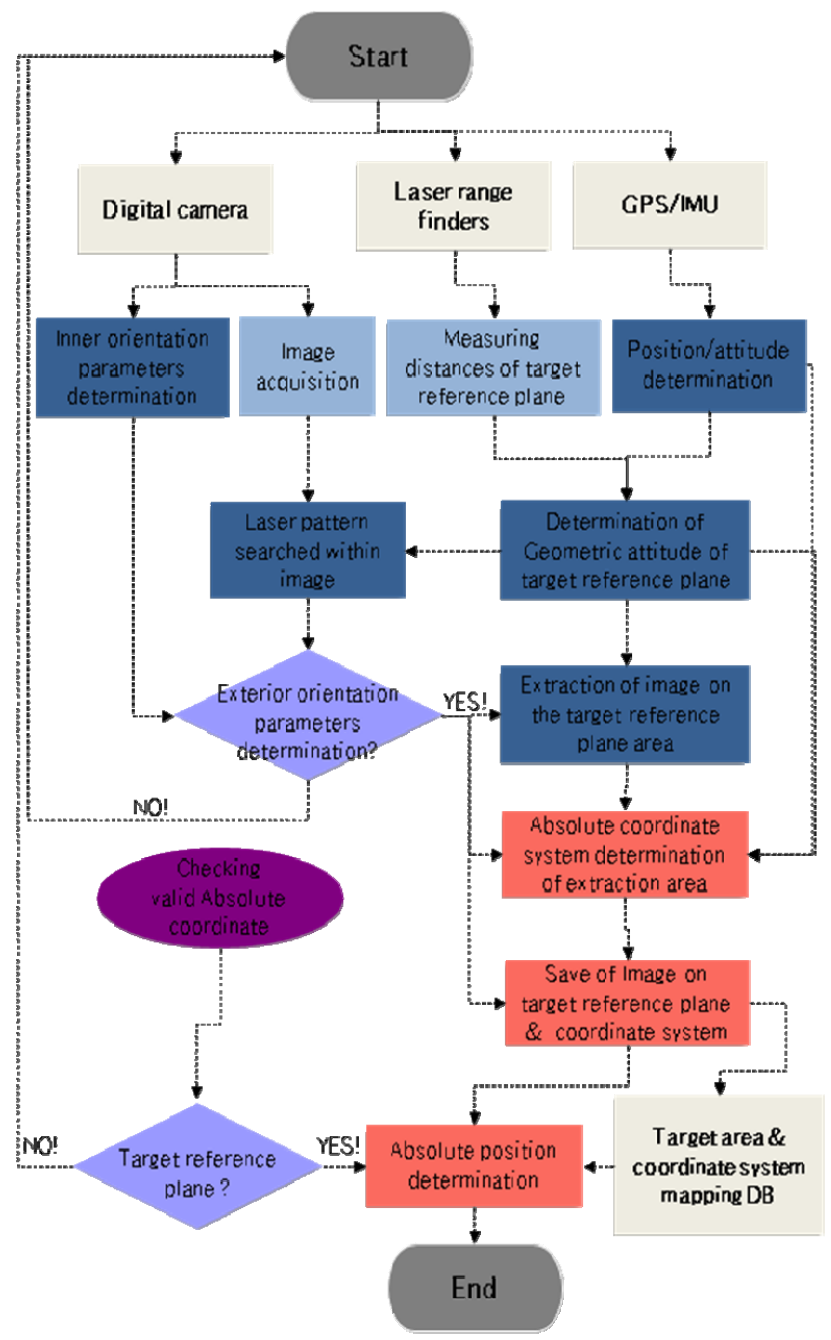

Fig. 5. The whole process to determine absolute position of single vision using laser range finders

\section{CONCLUSION}

In this research, we extracted the geometric relationship between the ground and images using sensor position, attitude, and distances obtained from laser ranging. We subsequently applied virtual model space assuming the road surface is flat and road sides are vertical planes. This approach enables estimation of size and position of a $3 \mathrm{D}$ object by extracting the absolute position from single vision. Because the proposed method can find the absolute position from a single image by generating a model for a target reference plane, an expensive and complicated system is not necessary. Moreover, the work process can be reduced by minimizing separate post-processing. In the future, if parallel processing is applied based on a graphic processor, real-time absolute positioning and $3 \mathrm{D}$ object extraction are expected to be available for application to a real-time mapping system. It is expected that the real-time absolute positioning technique can be used for various spatial information construction such as underwater mapping, road facility investigation and real-time fusion of road and 3D object information.

\section{ACKNOWLEDGMENT}

This research was supported by a grant from Cutting-Edge Urban Development-Korean Land Spatialization Research Project funded by Ministry of Land, Transport and Maritime Affairs (06KLSGB01) and by the supporting project to educate GIS experts funded by the Ministry of Land, Transport and Maritime Affairs of the Government of Korea.

\section{REFERENCES}

[1] D. Jeong, "A Comparative Study on the 3D Positioning Methods by CCD Images of The Mobile Mapping System," The Journal of GIS Association of Korea, vol. 15 no. 2, pp. 169-180, April 2007.

[2] T. Oh and I. Lee, "Determinnation of 3D object coordinates from overlapping omni-directional images acquired by a mobile mapping system," Korean Society of Surveying Geodesy Photogrammetry, vol. 28, no. 4, pp. 305-315, June 2010.

[3] M. Aly, "Real time detection of lane markers in urban streets," in Proc. Intelligent Vehicles Symposium, 2008 IEEE, 2008, pp. 7-12.

[4] S. Tuohy, D. O’Cualain, E. Jones, and M. Glavin, “Distance determination for an automobile environment using inverse perspective mapping in OpenCV, " in Proc. Irish Signals and Systems Conference 2010, 2010.

[5] S. G. Yoo and K. T. Chong, "Implementation of the SLAM System Using a Single Vision and Distance Sensors," Journal of the Institute of Electronics Engineers of Korea, vol. 45 no. 6, pp. 149-156, November 2008 .

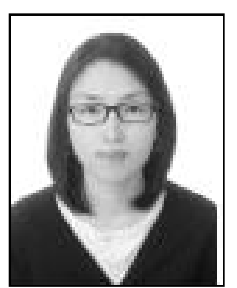

H. S. Woo was born in Seoul, Korea on February 1978. She received the M.S degree in geo-informatic engineering from Inha University, Incheon, Korea, in 2009.

She is currently pursuing the $\mathrm{Ph} . \mathrm{D}$. degree in geo-informatic engineering at Inha University, working on research project for Cutting-Edge Urban Development-Korean Land Spatialization which funded by Ministry of Land, Transport and Maritime Affairs. Her current research interests include photogrammetry, mobile mapping, u-GIS and spatial information determination.

Ms. Woo is a member of Korea Society of Surveying Geodesy Photogrammetry and Cartography.

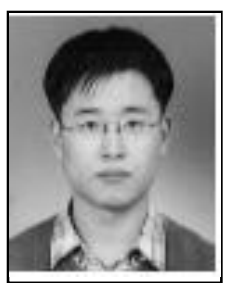

K. S. Kwon was born on January 1971. He received the M.S degree in geo-informatic engineering from Inha University, Incheon, Korea, in 1999.

$\mathrm{He}$ is currently pursuing the Ph.D. degree in geo-informatic engineering at Inha University. He is technical director in Underwater Survey Technology 21, Ltd. His current research interests include ocean space information, mobile mapping and autonomous control system.

Mr. Kwon is a member of Korea Society of Surveying Geodesy Photogrammetry and Cartography.

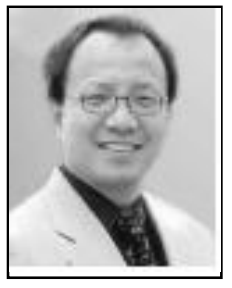

B. G. Kim was born on September 1955. He received the $\mathrm{Ph} . \mathrm{D}$. degree in geology and geophysics from University of Wisconsin-Madison, in 1989.

$\mathrm{He}$ was in Brunson Instrument Company as Photogrammetric System engineer from 1989 to 1990 and was in accelerator laboratory of Postech as the institute's director from 1990 to 1993 . He is now a professor in the Dept. of Geo-informatic Eng., Inha University, Incheon, KOREA and the leader of Korean Land Spatialization Group.

Dr. Kim was awarded The Baush \& Lomb Photogrammetric Award and The Robert E. Altenhofen Memorial Scholarship from ASPRS in 1987 and 1989 , respectively. 